\title{
Reconfiguration of Brain Network Architectures between Resting-State and Complexity-Dependent Cognitive Reasoning
}

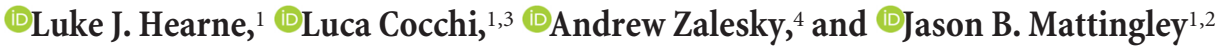 \\ ${ }^{1}$ Queensland Brain Institute and ${ }^{2}$ School of Psychology, The University of Queensland, St. Lucia, Queensland 4072, Australia, ${ }^{3}$ QIMR Berghofer Medical \\ Research Institute, Herston, Queensland 4006, Australia, and ${ }^{4}$ Melbourne Neuropsychiatry Centre, University of Melbourne, Melbourne, Victoria 3010, \\ Australia
}

Our capacity for higher cognitive reasoning has a measurable limit. This limit is thought to arise from the brain's capacity to flexibly reconfigure interactions between spatially distributed networks. Recent work, however, has suggested that reconfigurations of taskrelated networks are modest when compared with intrinsic "resting-state" network architecture. Here we combined resting-state and task-driven functional magnetic resonance imaging to examine how flexible, task-specific reconfigurations associated with increasing reasoning demands are integrated within a stable intrinsic brain topology. Human participants (21 males and 28 females) underwent an initial resting-state scan, followed by a cognitive reasoning task involving different levels of complexity, followed by a second resting-state scan. The reasoning task required participants to deduce the identity of a missing element in a $4 \times 4$ matrix, and item difficulty was scaled parametrically as determined by relational complexity theory. Analyses revealed that external task engagement was characterized by a significant change in functional brain modules. Specifically, resting-state and null-task demand conditions were associated with more segregated brain-network topology, whereas increases in reasoning complexity resulted in merging of resting-state modules. Further increments in task complexity did not change the established modular architecture, but affected selective patterns of connectivity between frontoparietal, subcortical, cingulo-opercular, and default-mode networks. Larger increases in network efficiency within the newly established task modules were associated with higher reasoning accuracy. Our results shed light on the network architectures that underlie external task engagement, and highlight selective changes in brain connectivity supporting increases in task complexity.

Key words: complexity; connectivity; fMRI; modularity; network; reasoning

Significance Statement

Humans have clear limits in their ability to solve complex reasoning problems. It is thought that such limitations arise from flexible, moment-to-moment reconfigurations of functional brain networks. It is less clear how such task-driven adaptive changes in connectivity relate to stable, intrinsic networks of the brain and behavioral performance. We found that increased reasoning demands rely on selective patterns of connectivity within cortical networks that emerged in addition to a more general, taskinduced modular architecture. This task-driven architecture reverted to a more segregated resting-state architecture both immediately before and after the task. These findings reveal how flexibility in human brain networks is integral to achieving successful reasoning performance across different levels of cognitive demand.

\section{Introduction}

Humans are unparalleled in their ability to reason and solve complex problems in the service of goal-directed behavior (Penn et

Received Feb. 20, 2017; revised July 13, 2017; accepted July 23, 2017.

Author contributions: L.J.H., L.C., and J.B.M. designed research; L.J.H. and L.C. performed research; L.J.H., L.C., and A.Z. analyzed data; L.J.H., L.C., A.Z., and J.B.M. wrote the paper.

This work was supported by the Australian Research Council (ARC) Special Research Initiatives Science of Learning Research Centre (SR120300015), and the ARC Centre of Excellence for Integrative Brain Function (ARC Centre Grant CE140100007). J.B.M. was supported by an ARC Australian Laureate Fellowship (FL110100103). L.C. was supported by a National Health and Medical Research Council (NHMRC) grant (APP1099082). A.Z. was supported by an NHMRC Career Development Fellowship (GNT1047648). L.J.H. was supported by an Australian Postgraduate Award. We thank Oscar Jacoby and Zoie Nott for data-collection assistance, Tong Wu for assistance with imaging al., 2008; Johnson-Laird, 2010). Nevertheless, our ability to reason successfully is limited by the complexity of the task at hand (Halford et al., 1998, 2005). Increasing reasoning demands are supported by the flexible reconfiguration of large-scale functional brain networks (Cocchi et al., 2013, 2014), but recent work

analysis, and Dr. Kieran O'Brien, Associate Professor Markus Barth, and Dr. Steffen Bollmann for performing the sequence optimizations.

The authors declare no competing financial interests.

Correspondence should be addressed to Luke J. Hearne, Queensland Brain Institute, The University of Queensland, St. Lucia, Queensland 4072, Australia. E-mail: I.hearne@uq.edu.au.

D0I:10.1523/JNEUROSCI.0485-17.2017

Copyright $\odot 2017$ the authors $\quad 0270-6474 / 17 / 378399-13 \$ 15.00 / 0$ 
has demonstrated that such reconfigurations are relatively modest and occur within a preserved global network architecture (Cole et al., 2014; Krienen et al., 2014). Here we assessed changes in functional brain architecture induced by engagement in a complex reasoning task, as well as changes in communication across regions with parametric increases in reasoning complexity. To do so, we used high-field functional magnetic resonance imaging (fMRI) to measure brain activity at rest and during performance of a behavioral task in which task complexity was manipulated parametrically.

Higher cognitive functions are supported by the adaptive reconfiguration of large-scale functional networks (Bassett et al., 2011; Cole et al., 2013; Braun et al., 2015; Cohen and D'Esposito, 2016; Yue et al., 2017). Previous empirical and theoretical work suggests that a multitude of complex tasks are related to activity and communication within and between select frontoparietal, cingulo-opercular, and default-mode networks (Knowlton et al., 2012; Cocchi et al., 2014; Hearne et al., 2015; Crittenden et al., 2016; Bolt et al., 2017). Such networks are flexible and tend to increase their functional relationship in line with task demands across a wide range of domains, including reasoning (Cocchi et al., 2014), working memory (Vatansever et al., 2017), and decision making (Cole et al., 2013).

Recent empirical work has shown that task-induced network reconfigurations are modest when compared with intrinsic, "resting-state" networks (Cole et al., 2014; Krienen et al., 2014). For example, Cole and colleagues reported a matrix-level correlation between rest and task states of $r=0.90$ (on average $38 \%$ of connections demonstrated change, with an average change of $r=$ 0.04). Likewise, it is now apparent that task-induced activity can be well predicted and modeled from resting-state data alone (Cole et al., 2016; Tavor et al., 2016). These results suggest that while behaviorally meaningful, selective task-induced reconfigurations occur against a backdrop of stable, large-scale networks that support diverse cognitive functions (Power et al., 2011; Crossley et al., 2013). An important unresolved question is how selective, "flexible" task-driven reconfigurations emerge among "stable" intrinsic brain topology. Moreover, it is critical to understand how such global and selective changes are related to behavior (Bolt et al., 2017; Mill et al., 2017).

To investigate this question, we measured functional brain networks at rest, as well as during several discrete levels of reasoning complexity. To systematically manipulate task complexity, we exploited relational complexity theory (Halford et al., 1998), which posits that the number of relations between variables quantifies the complexity of a problem, regardless of the domain of the original stimulus (e.g., semantic, spatial, etc.). Using this theoretical framework, it has been shown that increasing the number of relations imposes a quantifiable cognitive load (measured via reaction time and accuracy), and eventually results in a breakdown of the reasoning process (Halford et al., 2005). We collected 7 T fMRI data from 65 individuals while they undertook a nonverbal reasoning task known as the Latin Square Task (LST; Birney et al., 2006). During the task, participants solved problems with three discrete levels of difficulty, defined formally in terms of their relational complexity (binary, ternary, quaternary). In addition, just before the task, and again immediately afterward, participants underwent a resting-state scan. To examine network reconfigurations across rest and reasoning states, we used modularity to assess segregation and integration and global efficiency to assess changes in network communication. Further, to examine selective changes, we employed the network-based statistic to identify circumscribed changes in con- nectivity patterns (Zalesky et al., 2010), and related such network metrics to behavior.

\section{Materials and Methods}

Participants. Sixty-five healthy, right-handed participants undertook the current study, of whom 49 were included in the final analysis (mean, 23.35 years; SD, 3.6 years; range, $18-33$ years; 28 females). Four participants were excluded due to MR scanning issues, one participant was excluded due to an unforeseen brain structure abnormality, another participant was excluded due to low accuracy in the behavioral task (total score $>3$ SDs below the mean), and 10 participants were excluded due to excessive head movement (for head-movement exclusions, see Neuroimaging acquisition and preprocessing). Participants provided informed written consent to participate in the study. The research was approved by The University of Queensland Human Research Ethics Committee.

Experimental paradigm. Each participant completed two behavioral sessions and one imaging session. In the imaging session, participants underwent a resting-state scan, followed by three $12 \mathrm{~min}$ runs of the LST (described below), a structural scan, and finally a second resting-state scan (Fig. 1a).

In the two behavioral sessions, participants completed the Raven's Advanced Progressive Matrices (40 min time limit), which is a standard and widely used measure of fluid intelligence (Raven, 2000). Of the 49 participants included in the analysis, 43 also completed a conjunction visual search task in which they were instructed to report the orientation of a target letter " $\mathrm{L}$ " (rotated $90^{\circ}$ leftward or rightward) among "T" distractors in set sizes of 8,16 , or 24 items. The search cost was defined as the increase in reaction time between the smallest and largest set sizes. This task was chosen as a "low reasoning" counterpart to the Raven's Progressive Matrices to demonstrate the specificity of brain-behavior correlations, as described in detail in the Results.

Participants also completed a modified version of the LST (Birney et al., 2006; Birney and Bowman, 2009). The LST is a nonverbal relational reasoning paradigm in which reasoning complexity is parametrically varied with minimal working-memory demands (Halford et al., 1998; Birney et al., 2006). Each LST "puzzle" involves the presentation of a four-by-four matrix populated with a small number of geometric shapes (squares, circles, triangles, or crosses), blank spaces, and a single target, denoted by a yellow question mark (“?”; Fig. 1b). Participants were asked to solve for the target according to the following rule: each shape can only occur once in every row and once in every column (similar to the game of sudoku). Binary problems require integration of information across a single row or column. Ternary problems involve integration across a single row and column. Quaternary problems, the most complex, require integration of information across multiple rows and columns (Fig. 1b). Null trials involved presentation of an LST grid, but instead of a target question mark, an asterisk $\left({ }^{*}\right)$ was presented to cue the participant that no reasoning was required in this puzzle. The identity of the shapes that appeared in Null trials was random, but the number of shapes and their spatial locations were matched to those in the active LST trials. In total, 144 LST items were presented in the MR session across 16 blocks, with 36 items in each relational complexity condition; Null, Binary, Ternary, and Quaternary. Before the MR session, participants completed 20 practice trials of the LST (12 with corrective feedback). The visual angle subtended by the LST matrices was $\sim 7.7^{\circ}$, so that the entire stimulus fell within the parafoveal region of the visual field. Stimuli were projected onto a screen located at the head end of the MR scanner, and participants viewed the projected stimuli via a mirror mounted on the head coil.

Administration of all items was pseudorandomized such that no two items of the same complexity occurred sequentially, and each block had two problems from each level of complexity (Fig. 1c). Motor responses were counterbalanced across individuals, such that equal numbers of participants had the same shape-response mapping. Confidence ratings were used to determine participants' subjective feeling of success, and to identify any trials in which participants inadvertently disengaged from the task altogether (e.g., due to a momentary lapse of attention). A threepoint confidence scale indicated whether participants felt certain the problem had been answered correctly (4), felt unsure of their accuracy 
a

\begin{tabular}{|c|c|c|c|c|c|}
\hline $\begin{array}{c}\text { Pre-task } \\
\text { Rest }\end{array}$ & $\begin{array}{c}\text { Task } \\
\text { run 1 }\end{array}$ & $\begin{array}{c}\text { Task } \\
\text { run 2 }\end{array}$ & $\begin{array}{c}\text { Task } \\
\text { run 3 }\end{array}$ & T1 & $\begin{array}{c}\text { Post-task } \\
\text { Rest }\end{array}$ \\
\hline
\end{tabular}

b
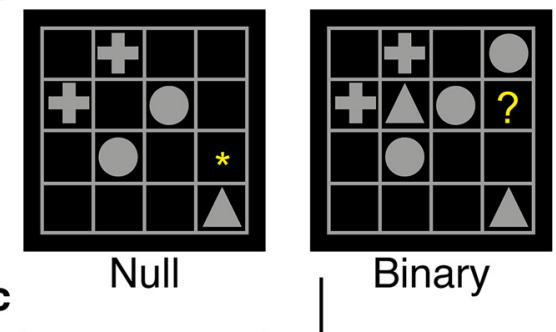

Binary

C

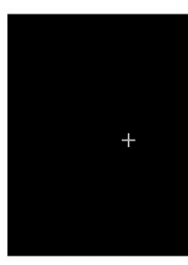

$4000 \mathrm{~ms}$ $\pm 586 \mathrm{~ms}$

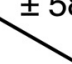

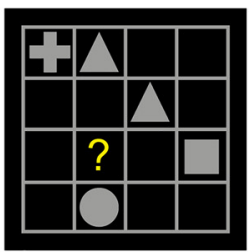

Ternary
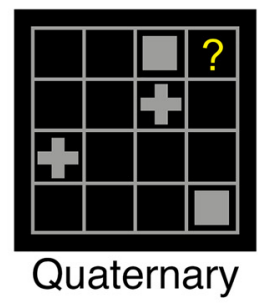

Imaging data were preprocessed using an adapted version of Matlab (MathWorks, RRID: SCR_001622) toolbox Data Processing Assistant for Resting-State fMRI (DPARSF V 3.0; ChaoGan and Yu-Feng, 2010; RRID: SCR_002372). Both resting-state and task data were preprocessed with the same pipeline (except where noted). DICOM (Digital Images and Communications in Medicine) images were first converted to Nifti format and realigned. T1 images were reoriented, skull-stripped [FMRIB (Oxford Centre of Functional MRI of the Brain) Software Library Brain Extraction Tool (FSL BET); RRID: SCR_009472], and coregistered to the Nifti functional images using statistical parametric mapping (SPM8; RRID: SCR_007037) functions. Segmentation and the DARTEL (diffeomorphic anatomical registration through exponentiated lie algebra) algorithm were used to improve the estimation of non-neural signal in subject space and the spatial normalization (Ashburner, 2007). From each gray matter voxel, the following signals were regressed: undesired linear trends, signals from the six head-motion parameters (three translation, three rotation), white matter, and CSF (estimated from single-subject masks of white matter and CSF). The CompCor method (Behzadi et al., 2007) was used to regress out residual signal unrelated to neural activity (i.e., five principal components derived from noise regions-of-interest in which the time series data were unlikely to be modulated by neural activity). Global signal regression was not performed due to the ongoing controversy associated with this step (Saad et al., 2012; Caballero-Gaudes and Reynolds, 2017). This choice may increase motion artifacts in the data (Ciric et al., 2017). For this reason, we employed a strict head-motion censoring approach (see below). Single-subject functional images were subsequently normalized and smoothed using DARTEL $\left(4 \mathrm{~mm}^{3}\right)$. Data processing steps also involved filtering $(0.01-0.15$ $\mathrm{Hz})$ at a low-frequency component of the BOLD signal known to be sensitive to both resting-state and task-based functional connectivity (Sun et al., 2004), therefore allowing comparison of both resting-state and task data.

Head movement. Participants with head displacement $>3 \mathrm{~mm}$ in $>5 \%$ of volumes in any

(3), or felt certain the problem had been answered incorrectly (2). On the far left (demarcated by a vertical line; Fig. 1c) was an additional "inattention" rating point (1) that participants were instructed to select if they felt they had not attempted to solve the problem due to a momentary lapse of attention, fatigue, or other factors. This response was used to separate incorrect choices arising from failures in reasoning, from those due to nonspecific "off-task" mind wandering (Smallwood and Schooler, 2015).

Neuroimaging acquisition and preprocessing. At the Centre for Advanced Imaging, The University of Queensland, imaging data were collected using a 7 T Siemens MR scanner fitted with a 32-channel head coil. For both resting-state and task fMRI, whole-brain echo-planar images were acquired using a multiband sequence (acceleration factor, 5; Moeller et al., 2010). In each of the two resting scans, 1050 volumes were collected ( $\sim 10 \mathrm{~min}$ each). In the each of the three runs of the task, 1250 volumes were collected ( $\sim 12 \mathrm{~min}$ each $)$ with the following parameters: voxel size, $2 \mathrm{~mm}^{3}$; TR $=586 \mathrm{~ms} ; \mathrm{TE}=23 \mathrm{~ms}$; flip angle, $40^{\circ}$; FOV, 208 $\mathrm{mm} ; 55$ slices. Structural images were also collected to assist functional data preprocessing. These images were acquired using the following parameters: MP2RAGE sequence; voxel size, $0.75 \mathrm{~mm}^{3}$; TR $=4300 \mathrm{~ms}$; $\mathrm{TE}=3.44 \mathrm{~ms} ; 256$ slices (Fig. 1a). one scan were excluded. In addition to gross head movement, it has also been shown that functional connectivity can be influenced by small volume-to-volume "micro" head movements (Van Dijk et al., 2012; Power et al., 2014). To ensure micro-head-movement artifacts did not contaminate our findings, both resting-state and task-based data with frame-to-frame displacements of $>0.40 \mathrm{~mm}$ were censored (Power et al., 2014 ). Participants with $<85 \%$ of data remaining in any condition were excluded.

Functional connectivity network construction. For each subject, regionally averaged time series were extracted for 264 spheres of $5 \mathrm{~mm}$ radius sampled across cortical and subcortical gray matter. Spheres were positioned according to an existing brain parcellation, based on task activations induced by a wide range of behavioral tasks (Power et al., 2011). This parcellation and associated network definitions were generated from a large cohort of participants $(N>300)$, and has the advantage of being independent of the imaging data obtained in the current study.

For both sets of resting-state data (Pre-task and Post-task), functional connectivity was estimated using a temporal Pearson correlation between each pair of time series (Zalesky et al., 2012). This resulted in a 
$264 \times 264$ connectivity matrix for each subject. For the task-based functional connectivity analyses, we used a regression approach (Cole et al., 2014) rather than psychophysiological interactions (PPIs) as others have used (McLaren et al., 2012; Cocchi et al., 2014; Gerchen et al., 2014). We opted for this approach rather than PPIs due to our interest in assessing connectivity across both rest and task states. For each brain region of interest, a task regressor composed of the condition onsets modeled as boxcar functions convolved with a canonical hemodynamic response function was regressed from the time series. This step was taken to remove variance associated with task-related coactivation (Cole et al., 2014). Then, after accounting for the hemodynamic lag, the residual time series from each $5 \mathrm{~s}$ reasoning period was concatenated to form in each brain region a condition-specific time series of interest. A Pearson correlation was performed on the resulting regional time series for each condition separately, resulting in a 4 (condition) $\times 264 \times 264$ connectivity matrix for each subject. Finally, both resting-state and task-based matrices were converted to $z$ scores. Analysis decisions such as $z$ normalization and thresholding were employed so as to be consistent with previous, related work aimed at assessing dynamic reconfiguration of connectivity patterns as a function of task demands (Cole et al., 2014; Power et al., 2011). Such choices do, however, affect the resulting graph metrics (Rubinov and Sporns, 2011). Thus, unless otherwise noted [see network-based statistic (NBS) analysis, below], weighted graphs of proportional densities from the top $5 \%$ to the top $30 \%$ of connections were considered for analysis. Such network densities have been shown to provide robust functional brain-network characterizations (Garrison et al., 2015) and are similar to those used in previous, related work (Power et al., 2011).

Analysis overview. We undertook three complementary analyses to identify functional network reorganization due to increasing relational complexity. First, we calculated and compared community partitions that arose in each of the resting-state and task conditions. Following this, we performed an analysis to identify changes in connectivity associated with performance of the LST using the NBS (Zalesky et al., 2010), a sensitive statistical tool that controls for type-I error at the network level. To assess the functional and behavioral impact of the connectivity changes identified in the previous two analyses, we calculated changes in global efficiency (Achard and Bullmore, 2007) for each functional module detected. Moreover, to assess the behavioral implications of the observed network changes, we correlated metrics of changes in module efficiency with performance accuracy on the LST. When appropriate, nonparametric statistics were used for repeated-measures comparisons (Friedman test), follow-up tests (Wilcoxon signed rank), and measures of effect size (Kendall's coefficient of concordance, $W$ ).

Community detection. A module is a group of nodes in a graph that contains stronger connections within module than expected in an appropriate random network null model. A modularity partition represents the subdivision of a graph into nonoverlapping modules (Fortunato, 2010). The degree of modularity in a network can be characterized by the $Q$ index (Newman and Girvan, 2004), which represents the density of within-module connections relative to an appropriate random network null model. The aim of community detection is to isolate a module partition that maximizes $Q$ according to the following equation:

$$
Q(\gamma)=\frac{1}{2 m} \sum_{i j}\left[a_{i j}-\gamma p_{i j}\right] \delta\left(\sigma_{i} \sigma_{j}\right)
$$

This is the modularity equation, where $a_{i j}$ represents the weight of the edge between $i$ and $j, p_{i j}=\frac{k_{i} k_{j}}{2 m}$ represents the expected number of links according to the so-called configuration null model (Newman et al., 2001), where $k_{\mathrm{i}}$ is the degree of node $i$; in this case the null preserves the node degree while forming connections at random. Meanwhile, $2 m$ represents the total number of connections in the network; $\sigma_{i}$ denotes the community to which node $i$ is assigned; and the Kronecker $\delta$ function, $\delta\left(\sigma_{i} \sigma_{j}\right)$, is 1 if $\sigma_{i}=\sigma_{j}$ and 0 if otherwise. Finally, $\gamma$ is the resolution parameter; when $\gamma<1$, larger communities are resolved; if $\gamma>1$, smaller communities are resolved.

In the present study, modules were identified using the Louvain greedy algorithm (Blondel et al., 2008) implemented in the Brain Connectivity Toolbox (BCT; Rubinov and Sporns, 2010; RRID: SCR_004841). The resolution parameter was set to unity $(\gamma=1)$. Testing across several levels of $\gamma$ showed consistent results. For clarity, we highlight the BCT scripts used throughout Materials and Methods. There are multiple possible module partitions that maximize $Q$ for each graph, resulting in community assignments that vary across each run of the algorithm (Good et al., 2010; Sporns and Betzel, 2016). To resolve this variability, we used a consensus approach (Lancichinetti and Fortunato, 2012), whereby module partitions are calculated a number of times $\left(10^{3}\right.$ iterations, for each participant and condition) and used to calculate an agreement matrix (agreement.m). The agreement matrix represents the tendency for each pair of nodes to be assigned to the same module across iterations. Finally, the agreement matrix was subjected to an independent module partitioning (consensus_und.m), resulting in an individual-level module partition for each participant in each condition. In this step, the resolution parameter was also set to unity $(\tau=1)$, representing the level at which the agreement matrix was thresholded before being subjected to the consensus procedure. For example, $\tau=1$ thresholds the matrix such that only nodes consistently partitioned into the same community across all permutations are included. Testing across several levels of $\tau$ showed consistent results. A general community structure including motor-sensory, auditory, visual, default-mode, and frontoparietal/cingulo-opercular modules was entered into the algorithm as the initial community partition. In our data, this choice decreased computation time, presumably because the initial community structure was associated with a $Q$ value that was close to the true maximum. Module reconfiguration results were replicated using different community affiliation priors, including variations of the original community partitions (Power et al., 2011; Cole et al., 2013) and purely data-driven methods (i.e., no community affiliation input).

The procedure for group-level modular decomposition was implemented in a similar fashion to the individual-level decompositions described above. The critical difference was that instead of creating an individual-level agreement matrix, the agreement matrix represented the tendency for each pair of nodes to be assigned to the same module across participants. The same consensus procedure followed, resulting in a single module partition for each condition for the group of 49 participants. Resolution parameters were kept identical to the previous individuallevel modularity analysis.

Significance testing for within-participant differences in modular structure. To investigate differences in the nodal composition of modules across conditions, we used the variation of information metric (VIn; Meilă, 2007), an information-theoretic measure of partition distance 
Table 1. Variation of information statistics

\begin{tabular}{|c|c|c|c|c|c|c|c|c|c|c|c|c|}
\hline \multirow[b]{3}{*}{ Contrast } & \multicolumn{12}{|c|}{ Network density } \\
\hline & \multicolumn{2}{|l|}{$5 \%$} & \multicolumn{2}{|l|}{$10 \%$} & \multicolumn{2}{|l|}{$15 \%$} & \multicolumn{2}{|l|}{$20 \%$} & \multicolumn{2}{|l|}{$25 \%$} & \multicolumn{2}{|l|}{$30 \%$} \\
\hline & VIn & $p$ & VIn & $p$ & VIn & $p$ & VIn & $p$ & Vln & $p$ & VIn & $p$ \\
\hline Post-task rest & 0.056 & 0.878 & 0.065 & 0.863 & 0.109 & 0.656 & 0.098 & 0.836 & 0.124 & 0.552 & 0.092 & 0.944 \\
\hline Null & 0.146 & 0.013 & 0.139 & 0.077 & 0.124 & 0.397 & 0.189 & $0.009^{*}$ & 0.198 & $0.005^{*}$ & 0.186 & 0.029 \\
\hline Binary & 0.148 & $0.006^{*}$ & 0.179 & $0.001^{*}$ & 0.218 & $0.001^{*}$ & 0.219 & $<0.001^{*}$ & 0.216 & $0.007^{*}$ & 0.209 & 0.023 \\
\hline \multicolumn{13}{|l|}{ Post-task rest } \\
\hline Null & 0.117 & 0.231 & 0.154 & 0.019 & 0.098 & 0.81 & 0.153 & 0.084 & 0.185 & 0.015 & 0.191 & 0.018 \\
\hline Binary & 0.128 & 0.099 & 0.185 & $0.001^{*}$ & 0.176 & 0.033 & 0.181 & 0.074 & 0.212 & $0.01^{*}$ & 0.203 & 0.018 \\
\hline Ternary & 0.16 & $0.006^{*}$ & 0.204 & $<0.001^{*}$ & 0.194 & $0.006^{*}$ & 0.203 & $0.003^{*}$ & 0.2 & 0.016 & 0.22 & $0.001^{*}$ \\
\hline Quaternary & 0.128 & 0.083 & 0.189 & $0.002^{*}$ & 0.195 & $0.005^{*}$ & 0.202 & $0.001^{*}$ & 0.2 & $0.005^{*}$ & 0.211 & $0.001^{*}$ \\
\hline \multicolumn{13}{|l|}{ Null } \\
\hline Ternary & 0.096 & 0.681 & 0.154 & 0.159 & 0.097 & 0.801 & 0.1 & 0.485 & 0.093 & 0.571 & 0.11 & 0.306 \\
\hline Quaternary & 0.038 & 0.998 & 0.121 & 0.375 & 0.086 & 0.91 & 0.111 & 0.507 & 0.106 & 0.484 & 0.108 & 0.387 \\
\hline \multicolumn{13}{|l|}{ Ternary } \\
\hline Quaternary & 0.082 & 0.747 & 0.084 & 0.894 & 0.041 & 1 & 0.051 & 0.986 & 0.061 & 0.943 & 0.051 & 0.969 \\
\hline
\end{tabular}

(partition_distance.m). To ascribe statistical significance to differences in partition structure, we used a repeated-measures permutation procedure to compare real VIn values to appropriate null distributions (Dwyer et al., 2014). Specifically, half of the participants' condition labels were randomly switched in the contrast of interest (e.g., Binary vs Ternary). This resulted in two new sets of individual-level module structures for the contrast (albeit with shuffled data). The shuffled module structures were then subjected to the previously used pipeline to generate group-level module partitions. Finally, VIn was used to quantify the difference between these partitions. This procedure was repeated $10^{4}$ times to build a null distribution for each contrast of interest, with which the real data were compared.

Pairwise functional connectivity analysis. The NBS (Zalesky et al., 2010; RRID: SCR_002454) was used to identify changes in pairwise functional connectivity at rest and during the task. For the first contrast, a paired $t$ test was performed between the Pre-task and Post-task resting-state data. For the second contrast, a one-way repeated-measures ANOVA was used to compare all four task states (Null, Binary, Ternary, Quaternary). For the analysis, unthresholded functional connectivity matrices were used as input into the NBS. Briefly, all possible pairs of connections $[(264 \times 263) / 2=34,716]$ were tested against the null hypothesis, endowing each connection with a test statistic, which was subsequently thresholded. Here an exploratory $F$ statistic of 20 (equivalent to a $t$ statistic of 4.47) was used as the threshold, though additional exploratory analyses showed that networks arising using higher or lower $t$ thresholds resembled the original results. This threshold was adopted because it allowed the detection of effects of medium size while discarding small or spurious effects. Familywise error (FWE)-corrected $p$ values were ascribed to the resulting networks using a null distribution obtained by 5000 permutations. Only components that survived a network-level threshold of $p<$ 0.001 (FWE corrected) were declared significant. This analysis allowed us to identify subnetworks that significantly increased or decreased their functional connectivity across relational reasoning task conditions, providing complementary results to the graph analyses.

Network efficiency analysis. Global efficiency is defined as the inverse of the average characteristic path length between all nodes in a network (Latora and Marchiori, 2001). Assuming that information follows the most direct path, global efficiency provides an index for parallel information transfer in a network (Rubinov and Sporns, 2010). In the context of functional brain networks, global efficiency is thought to be an index of increased capacity for information exchange (Achard and Bullmore, 2007). The link between indices of global efficiency and global neural information transfer is, however, not yet clear. Nevertheless, a number of studies have shown that high global brain-network efficiency can enhance neurophysiological (de Pasquale et al., 2016; Cocchi et al., 2017) and cognitive processes (Bassett et al., 2009; van den Heuvel et al., 2009; Shine et al., 2016).

Here we wanted to investigate differences in network communication within module, and determine how such difference might relate to behavior. To do so, we computed global efficiency for each participant, in each condition, for the three major modules identified in the initial modularity analysis (using efficiency_wei.m from the BCT). Importantly, matrix thresholding was performed after dividing the modules to ensure any efficiency effects were not due to differences in degree across modules. Finally, we computed the difference in efficiency between the mostdifficult and least-difficult conditions (i.e., Quaternary vs Null) and correlated this change in efficiency with overall accuracy scores on the LST.

Figures and visualization. Figures were generated with a combination of Matlab and on-line network visualization tools [alluvial diagram (http://www.mapequation.org/apps/MapGenerator.html) and the connectogram (http://immersive.erc.monash.edu.au/neuromarvl/].

\section{Results}

\section{Behavioral results}

A nonparametric Friedman test revealed a significant effect of reasoning complexity on both LST accuracy $\left(\chi^{2}=86.20\right.$, Kendall's $W=0.88, p<0.001)$ and reaction time $\left(\chi^{2}=63.71, W=0.65\right.$, $p<0.001$; Fig. 2). Bonferroni-corrected follow-up Wilcoxon signed-rank test comparisons revealed that accuracy was significantly higher for the Binary condition (mean, 34.96; SD, 1.04) than for both the Ternary condition (mean, 31.63; SD, 3.52; $z=$ 5.59; $p<0.001$ ) and the Quaternary condition (mean, 22.78; SD, $6.71 ; z=6.10 ; p<0.001$ ). Accuracy was also higher for Ternary items than for Quaternary items $(z=5.74, p<0.001)$. The reaction time results followed a similar pattern, such that responses were faster in the Binary condition (mean, $771.60 \mathrm{~ms}$; SD, 201.60 $\mathrm{ms}$ ) than in the Ternary (mean, $844.20 \mathrm{~ms}$; SD, $217.30 \mathrm{~ms} ; z=$ -4.62; $p<0.001$ ) and Quaternary (mean, $933.70 \mathrm{~ms}$; SD, 205.00 $\mathrm{ms} ; z=-5.83 ; p<0.001)$ conditions. Likewise, reaction times in 
a

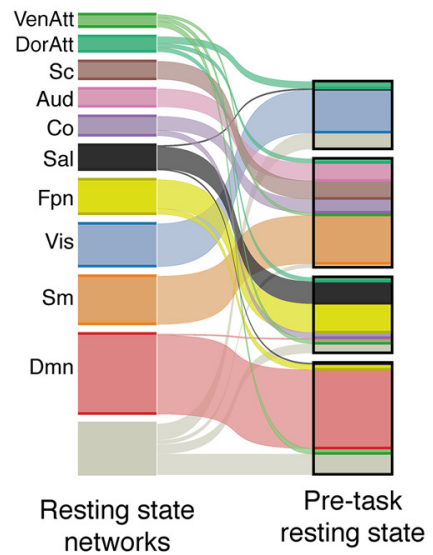

b

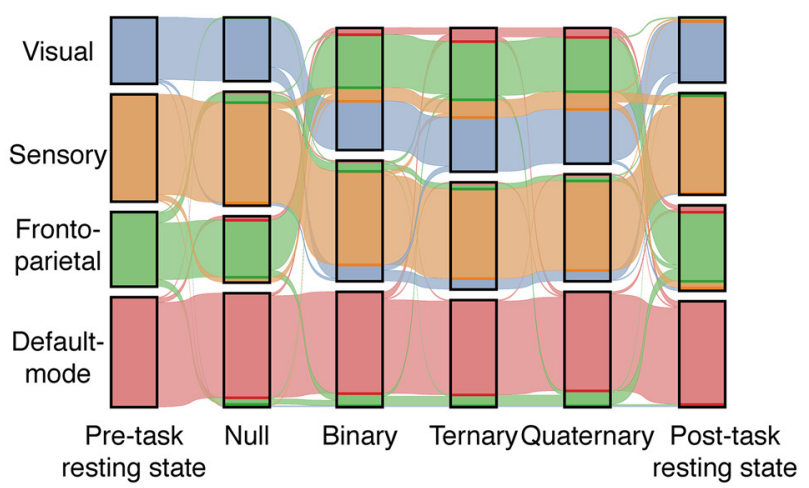

(Power et al., 2011)

C

- Visual $\odot$ Sensory $\odot$ Fronto-parietal $\bullet$ Default-mode

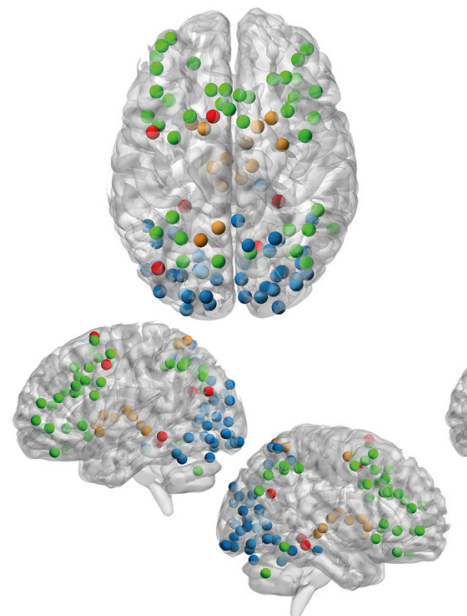

Task module 1

"Fronto-parietal-visual"

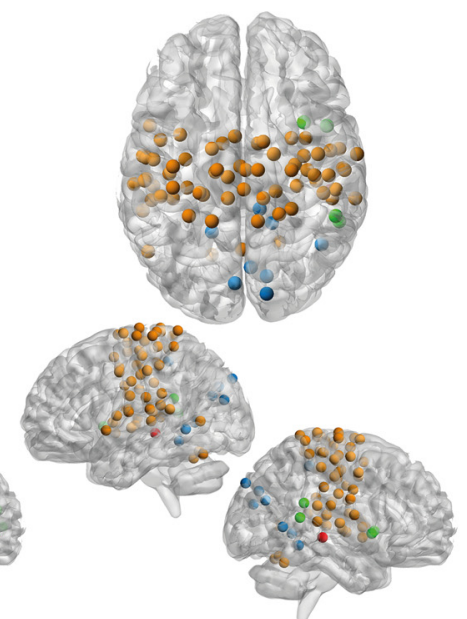

Task module 2

"Sensory"

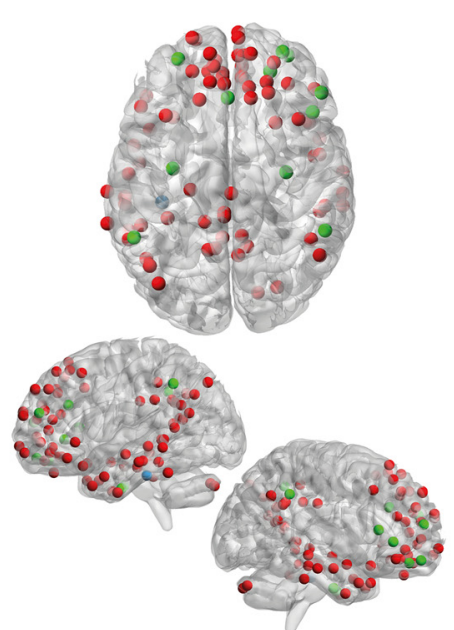

Task module 3 "Default-mode"

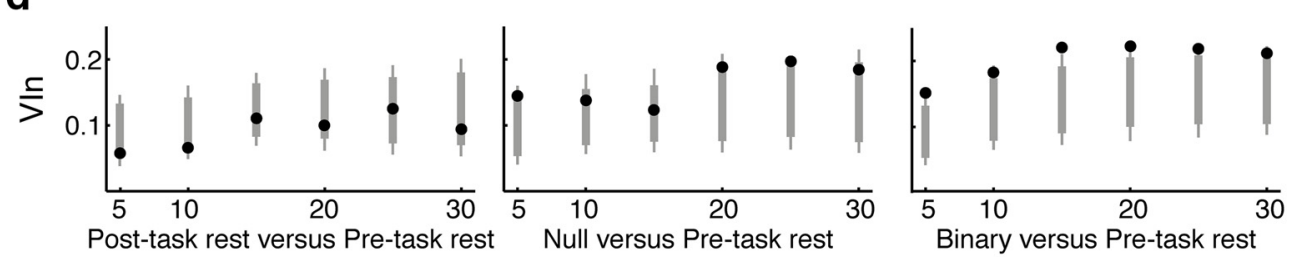

Network density (\%)

e

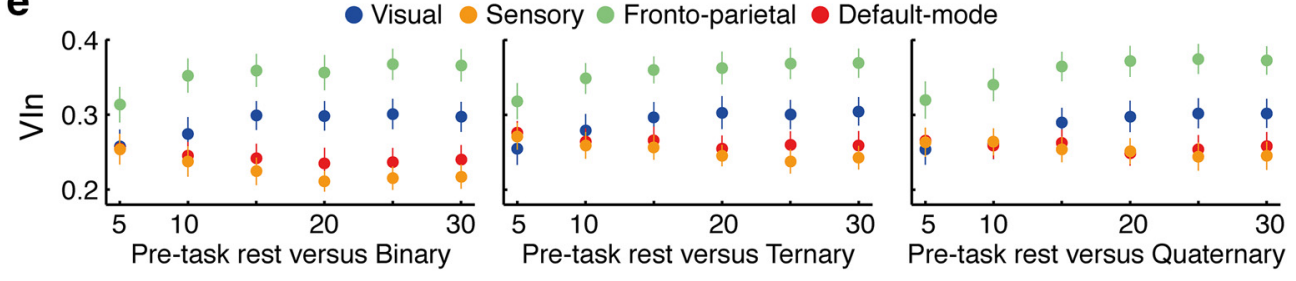

Network density (\%)

Figure 3. Modular structure as a function of reasoning complexity in the LST. $\boldsymbol{a}$, Alluvial "flow" demonstrating the network affiliations (Power et al., 2011) compared with the Pre-task resting-state (Rosvall et al., 2009). Each individual streamline represents a node in the network, colored by its original resting-state affiliation as shown on the left (Power et al., 2011). b, VenAtt, Ventral attention; DorAtt, dorsal attention; Sc, subcortical; Aud, auditory; Co, cingulo-opercular; Sal, salience; Fpn, fronto-parietal; Vis, visual; Sm, sensorimotor; Dmn, default-mode. Changes in modular structure across the experimental conditions. Visual and frontoparietal modules merged to form a task-related module during Binary, Ternary, and Quaternary conditions of the LST. Results for 15\% network density are shown, but statistics were performed across several thresholds. $\boldsymbol{c}$, Anatomical rendering of the task-related modules in the Quaternary condition. Each sphere is color-coded by its initial resting-state module allegiance. $\boldsymbol{d}$, VIn values (black markers) compared with a null distribution (gray markers: fifth- $95^{\text {th }}$ percentile in bold line; first- $99^{\text {th }}$ percentile shown in tails) for the three main contrasts across all network densities. Only the right-most contrast (Binaryvs Pre-task rest) showed a consistent difference between partitions.e, Comparison of VIn values across visual, sensory, frontoparietal, and default-mode modules in each task condition compared with rest across all network densities. The frontoparietal module was consistently more variable in relation to other modules. Error bars represent $95 \%$ confidence intervals. 
a

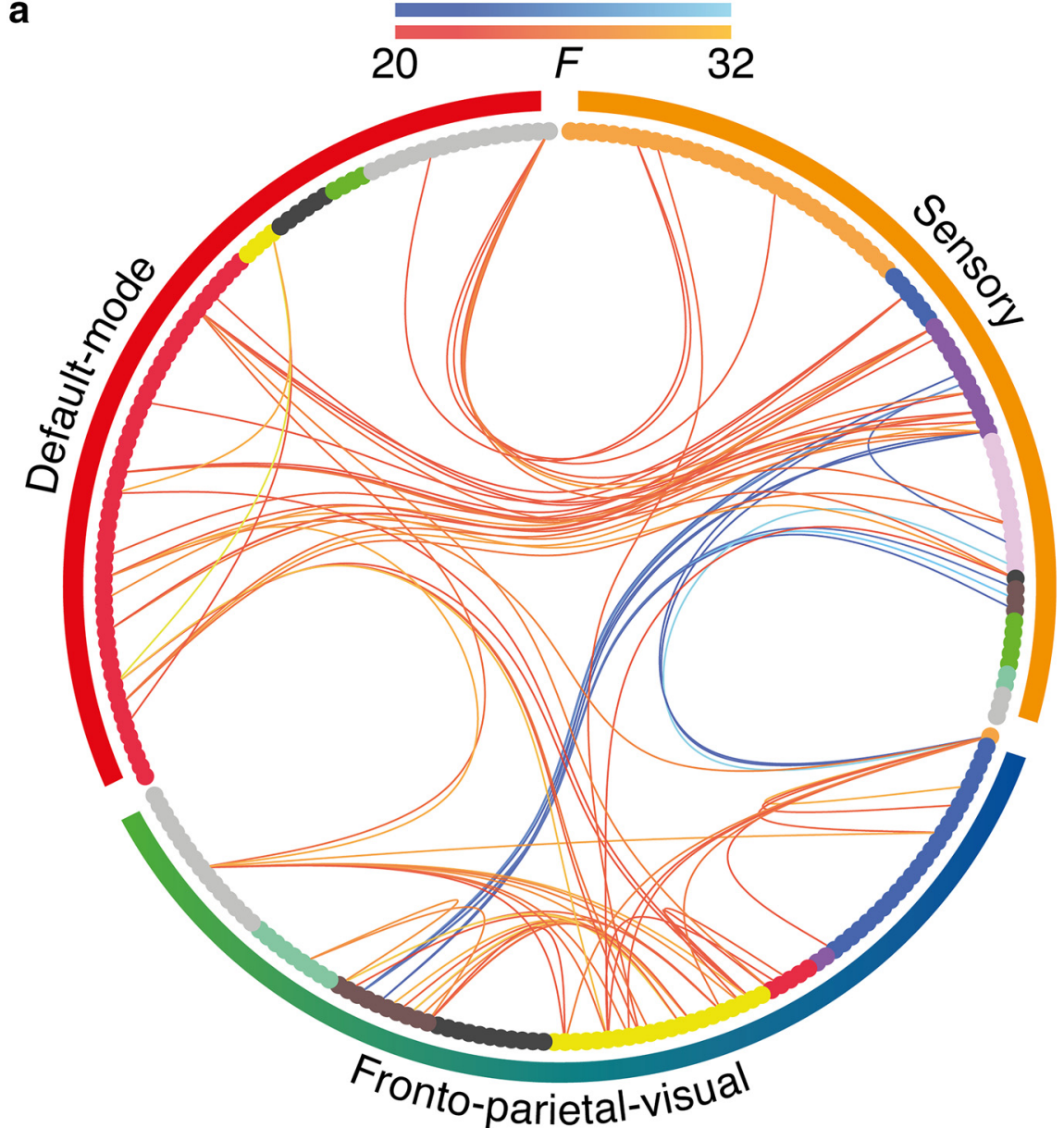

$\begin{array}{llll}\text { SSmotor } & \text { Cingulo-opercular } & \text { Salience } & \text { Ventral Attention } \\ \text { Visual } & \text { Default-mode } & \text { Subcortical } & \text { Dorsal Attention } \\ \text { Auditory } & \text { FPN } & \text { Other } & \end{array}$

b

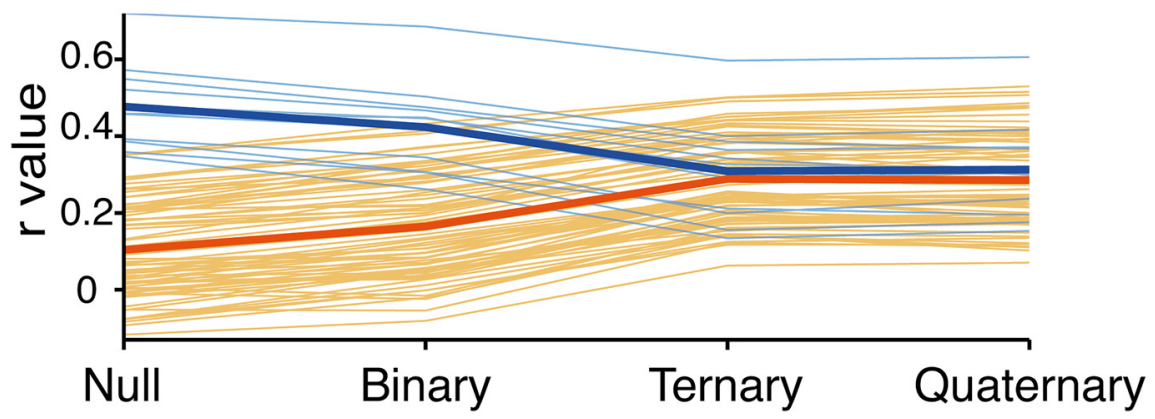

Figure 4. Change in pairwise functional connectivity associated with reasoning complexity. $\boldsymbol{a}$, Connectogram representation of significant changes in pairwisefunctional connectivitythatscaled with relational complexity. Edge colorsindicatedirection of correlation change acrossrelational complexity. Warm colors represent increases in connectivity and cool colors represent decreases in connectivity. Lighter colors represent higher $F$ statistics. Network nodes, plotted as circles, are colored by their initial resting-state networks (Power et al., 2011). Outside the connectogram, the colored bars represent the modules identified in the previous analysis of data from the Quaternary condition: sensory (orange), default-mode (red), and FPV modules (green-blue). $\boldsymbol{b}$, Each individual connection in the subnetwork (averaged across subjects) plotted as a function of reasoning complexity. Average values for positive and negative connections are shown as bold lines.

the Ternary condition were significantly faster than those in the Quaternary condition $(z=-4.79, p<0.001)$.

As expected, there was a significant positive correlation between scores on the Raven's Advanced Progressive Matrices, a measure of fluid intelligence, and overall accuracy on the LST $(r=0.44, p=0.002)$. By contrast, for the visual search task, there was no correlation between reaction time cost and LST score $(N=43, r=$ $-0.09, p=0.58$ ). A Steiger $z$ test (Lee and Preacher, 2013) demonstrated that these two correlations were significantly different from one another $(N=43, z=-2.90$, $p=0.003$ ), confirming that LST performance is linearly related to an established measure of fluid intelligence, but not to a widely used test of visual attention (Treisman and Gelade, 1980).

Participants' confidence was assessed on each trial. Importantly, we included an explicit rating for when participants had not attempted the reasoning problem due to an attention lapse, mind wandering, or fatigue. Averaging across all conditions, the mean number of such lapses was $<1$ of 36 trials (mean across conditions, 0.47 trials; SD across conditions, 0.98 trials). We can thus conclude that overall, participants were able to engage as instructed in cognitive reasoning across all three levels of relational complexity in the LST.

\section{Functional brain module reconfiguration}

Modularity analysis revealed four major modules in the baseline (Pre-task) resting-state. For clarity, these modules are represented in reference to Power and colleagues' (2011) initial network affiliations. The modules broadly correspond to the sensory, default-mode, visual, and frontoparietal networks.

VIn analysis (Meilă, 2007) revealed a significant difference in the community structure of Binary, Ternary, and Quaternary conditions compared with the Pretask resting-state (mean statistics across thresholds are reported in text; Table 1; VIn $=0.20, p=0.006$; VIn $=0.21, p=$ 0.001 ; VIn $=0.20, p=0.003$, respectively) and Post-task resting-state (VIn $=0.18$, $p=0.039 ; \mathrm{VIn}=0.20, p=0.005 ; \mathrm{VIn}=$ $0.19, p=0.016)$. The difference between rest and reasoning states was associated with the emergence of a single, conjoined frontoparietal-visual (FPV) module that was composed of several large-scale networks identified by Power et al. (2011). The transitory nature of the FPV module was confirmed by its switch back to its original configuration in the Post-task resting-state (i.e., after completion of the LST). Figure $3 b$ shows a representation of the reconfiguration of modules across experimental conditions. There was no significant difference between the Pre-task and Post-task resting-state community structure (VIn $=0.09, p=$ 0.788 ). There was also no consistent difference between the Pre- 
task and Post-task resting-state communities and the Null task condition (VIn $=0.16, p=0.08$; VIn $=0.15 p=0.196$, respectively). These main effects were broadly replicated across all thresholds tested (Fig. 3c).

Having established a difference in community structure, we sought to test the relative contribution of each module to the observed reconfiguration. To do so, we implemented a similar strategy to that of Braun and colleagues (2015), whereby VIn was calculated at the individual-community level for our nodes of interest, and compared using repeated-measures statistics. Thus, we compared visual, sensory, frontoparietal, and default-mode modules across all network densities for the Binary-Rest, TernaryRest, and Quaternary-Rest contrasts (conceptually similar to follow-up parametric statistics). Results revealed that the frontoparietal module had higher VIn values (i.e., larger differences in community structure) than visual (mean $p$ value across thresholds, $p<0.001)$, sensory $(p<0.001)$, and default-mode modules $(p<0.001$; Fig. $3 d)$ across all contrasts.

Finally, the index of modularity, $Q$, was compared across conditions. This index of modularity increases as more intramodular connections are found than expected by chance (Newman and Girvan, 2004). Nonparametric Friedman tests revealed a significant difference in $Q$ across conditions (mean statistic across thresholds, $\left.\chi^{2}=51.48, p<0.001\right)$. Bonferroni-corrected follow-up tests were performed to compare each task state (Null, Binary, Ternary, Quaternary) with each resting-state (Pre-task and Post-task). Results revealed that $Q$ was significantly lower in the Ternary (mean $Q=0.39$ ) and Quaternary conditions (mean $Q=0.39$ ) when compared with both Pre-task (mean $Q=0.44, z=$ 3.74, $z=4.07, p<0.001$ ) and Post-task resting-states (mean $Q=$ $0.45, z=4.49, z=4.52, p<0.001$ ). No effect was found when comparing Null or Binary conditions with rest. Complementing the observed changes in community structure, analysis of $Q$ scores highlights a significant reduction in modularity compared with the resting-state, but only in the task conditions that imposed higher demands on cognitive reasoning.

\section{NBS analysis}

To further refine our account of module reconfiguration, we assessed changes in whole-brain connectivity using the NBS ( $\mathrm{Za}$ lesky et al., 2010). In line with the result from the first analysis, a paired $t$ test between Pre-task and Post-task resting-states revealed no significant differences. Our second contrast, a oneway repeated-measures ANOVA was performed comparing all four reasoning complexity conditions (Null, Binary, Ternary, Quaternary).

A subnetwork comprising 63 nodes and 85 edges changed in response to reasoning complexity demands $(p<0.001$, FWE corrected at the network level; Fig. 4). Most edges within the subnetwork demonstrated increased functional connectivity ( $86 \%$ of edges; Fig. 4, warm colors), but a number of edges also demonstrated a decrease in positive correlations with increasing reasoning complexity (Fig. 4b). Consistent with our previous work on changes in functional connectivity during complex reasoning (Cocchi et al., 2014; Hearne et al., 2015), the network was largely composed of nodes encompassing frontoparietal (17\%), subcortical $(19 \%)$, cingulo-opercular $(12 \%)$, and default-mode networks (24\%; Power et al., 2011; Table 2). Moreover, nearly all edges $(95 \%)$ were across-network. Two further visual-parietal subnetworks were identified by the NBS, consisting of two and three nodes respectively (not visualized).
Table 2. Significant pairwise changes in functional connectivity associated with increasing relational complexity

\begin{tabular}{|c|c|c|c|c|c|c|}
\hline \multicolumn{3}{|l|}{$\overline{\mathrm{MNI}}$} & \multirow[b]{2}{*}{ RSN } & \multirow[b]{2}{*}{ Mod } & \multirow[b]{2}{*}{ Anatomy } & \multirow[b]{2}{*}{ Degree } \\
\hline$x$ & $y$ & $z$ & & & & \\
\hline-7 & -52 & 61 & Sshand & FPV & Precuneus & 12 \\
\hline 4 & -48 & 51 & Mem & FPV & Precuneus & 10 \\
\hline 47 & 10 & 33 & Fpn & FPV & Precentral gyrus & 7 \\
\hline-2 & -35 & 31 & Mem & Dmn & Middle cingulate cortex & 6 \\
\hline-34 & 3 & 4 & $\mathrm{C}_{0}$ & Sens & Middle insula & 6 \\
\hline 23 & 10 & 1 & Sc & FPV & Putamen & 6 \\
\hline 49 & 8 & -1 & Co & Sens & Superior temporal pole & 5 \\
\hline-11 & -56 & 16 & Dmn & Dmn & Calcarine gyrus & 5 \\
\hline-2 & 38 & 36 & Dmn & Dmn & Medial superior frontal gyrus & 5 \\
\hline-42 & -55 & 45 & Fpn & FPV & Inferior parietal cortex & 5 \\
\hline-45 & 0 & 9 & Co & Sens & Rolandic operculum & 4 \\
\hline 52 & -59 & 36 & Dmn & Dmn & Angular gyrus & 4 \\
\hline-35 & 20 & 51 & Dmn & Dmn & Middle frontal gyrus & 4 \\
\hline 9 & -4 & 6 & Sc & FPV & Thalamus & 4 \\
\hline-2 & -13 & 12 & Sc & FPV & Thalamus & 4 \\
\hline-47 & 11 & 23 & Fpn & FPV & Inferior frontal gyrus (operculum) & 4 \\
\hline-42 & 45 & -2 & Fpn & FPV & Inferior frontal gyrus (orbital) & 4 \\
\hline 54 & -28 & 34 & Co & Sens & Supramarginal gyrus & 3 \\
\hline 8 & -48 & 31 & Dmn & Dmn & Posterior cingulate cortex & 3 \\
\hline 13 & 30 & 59 & Dmn & Dmn & Superior frontal gyrus & 3 \\
\hline 11 & -39 & 50 & Sal & Sens & Middle cingulate cortex & 3 \\
\hline 15 & -77 & 31 & Vis & Sens & Cuneus & 3 \\
\hline-41 & 6 & 33 & Fpn & FPV & Precentral gyrus & 3 \\
\hline-53 & -49 & 43 & Fpn & Dmn & Inferior parietal cortex & 3 \\
\hline-44 & 2 & 46 & Fpn & FPV & Precentral gyrus & 3 \\
\hline 3 & -17 & 58 & Sshand & Sens & Supplementary motor area & 2 \\
\hline 65 & -33 & 20 & Aud & Sens & Superior temporal gyrus & 2 \\
\hline-51 & 8 & -2 & $\mathrm{Co}_{0}$ & Sens & Insula & 2 \\
\hline 15 & -63 & 26 & Dmn & FPV & Cuneus & 2 \\
\hline 11 & -54 & 17 & Dmn & Dmn & Precuneus & 2 \\
\hline 36 & 10 & 1 & Co & Sens & Insula & 2 \\
\hline-10 & -18 & 7 & Sc & FPV & Thalamus & 2 \\
\hline-22 & 7 & -5 & Sc & FPV & Putamen & 2 \\
\hline 43 & -78 & -12 & Vis & FPV & Inferior occipital cortex & 2 \\
\hline-42 & -60 & -9 & DorAtt & FPV & Inferior temporal gyrus & 2 \\
\hline-3 & 26 & 44 & Fpn & FPV & Superior frontal gyrus (medial) & 2 \\
\hline 44 & -53 & 47 & Fpn & FPV & Inferior parietal cortex & 2 \\
\hline 32 & 14 & 56 & Fpn & FPV & Middle frontal gyrus & 2 \\
\hline-40 & -19 & 54 & Sshand & Sens & Precentral gyrus & 1 \\
\hline 0 & -15 & 47 & Sshand & Sens & Middle cingulate cortex & 1 \\
\hline-10 & -2 & 42 & Co & Sens & Middle cingulate cortex & 1 \\
\hline-50 & -34 & 26 & Aud & Sens & Supramarginal gyrus & 1 \\
\hline 59 & -17 & 29 & Aud & Sens & Postcentral gyrus & 1 \\
\hline 37 & 1 & -4 & $\mathrm{Co}_{0}$ & Sens & Insula & 1 \\
\hline 6 & -59 & 35 & Dmn & Dmn & Precuneus & 1 \\
\hline-41 & -75 & 26 & Dmn & FPV & Middle occipital cortex & 1 \\
\hline 65 & -31 & -9 & Dmn & Dmn & Middle temporal gyrus & 1 \\
\hline 43 & -72 & 28 & Dmn & FPV & Middle occipital cortex & 1 \\
\hline-3 & 42 & 16 & Dmn & Dmn & Anterior cingulate cortex & 1 \\
\hline-16 & 29 & 53 & Dmn & Dmn & Superior frontal gyrus & 1 \\
\hline 2 & -24 & 30 & Mem & Dmn & Middle cingulate cortex & 1 \\
\hline 12 & 36 & 20 & Dmn & Dmn & Anterior cingulate cortex & 1 \\
\hline 6 & -24 & 0 & Sc & FPV & Thalamus & 1 \\
\hline 12 & -17 & 8 & Sc & FPV & Thalamus & 1 \\
\hline-5 & -28 & -4 & Sc & FPV & Lingual gyrus & 1 \\
\hline 31 & -14 & 2 & Sc & Sens & Putamen & 1 \\
\hline 29 & 1 & 4 & Sc & Sens & Putamen & 1 \\
\hline-31 & -11 & 0 & Sc & Sens & Putamen & 1 \\
\hline 15 & 5 & 7 & Sc & FPV & Pallidum & 1 \\
\hline 37 & -84 & 13 & Vis & FPV & Middle occipital cortex & 1 \\
\hline 37 & -81 & 1 & Vis & FPV & Middle occipital cortex & 1 \\
\hline-33 & -79 & -13 & Vis & FPV & Inferior occipital cortex & 1 \\
\hline 49 & -42 & 45 & Fpn & FPV & Inferior parietal cortex & 1 \\
\hline
\end{tabular}

Automated Anatomical Labeling atlas was used to define anatomical regions. RSN, Initial Power et al., 2011, resting-stat network affiliation; Mod, modules defined by modularity analysis in the Quaternary condition (i.e., Figure 3); $\mathrm{C}_{\text {, cingulo- }}$ opercular; Dmn, default-mode; Fpn, frontoparietal; Vis, visual; Sens, sensory; Sc, subcortical; Sal, salience; Aud, auditory; Mem, memory; Sshand, somatosensory hand; DorAtt, dorsal attention; FPV, fronto-parietal-visual. 
a

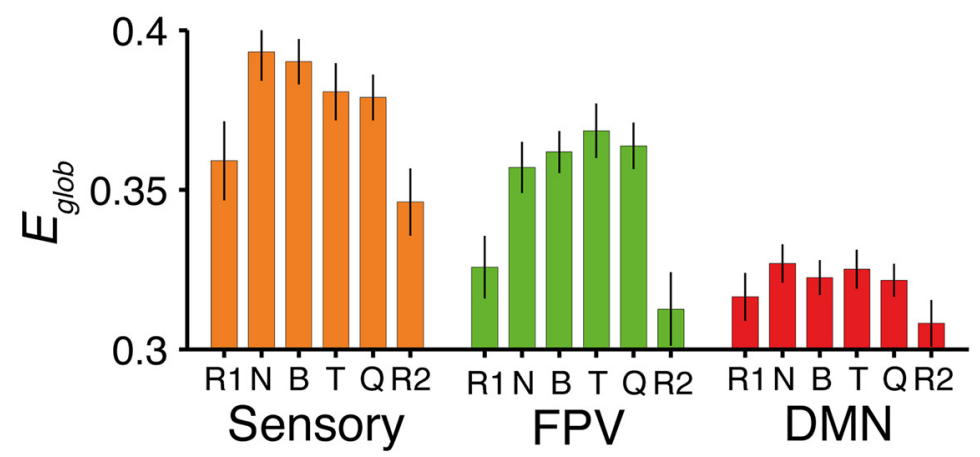

b

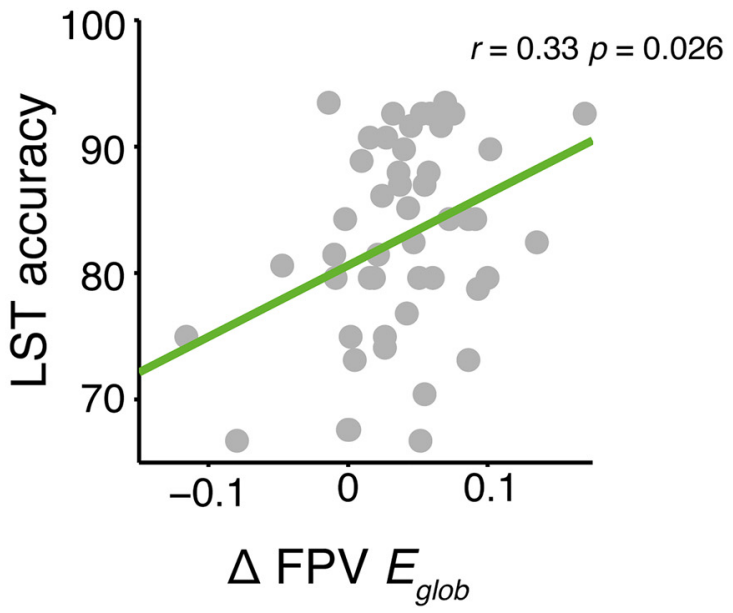

Figure 5. Changes in global network efficiency $\left(E_{\text {glob }}\right)$ across the identified reasoning task modules. $\boldsymbol{a}$, Global network efficiency levels within each module across experiment conditions. Error bars represent $95 \%$ confidence intervals. R1, Pre-task rest; B, Binary; T, Ternary; $Q$, Quaternary; R2, Post-task rest. $\boldsymbol{b}$, Correlation between accuracy in the LST and changes in FPV module efficiency during the task. Changes in network efficiency were correlated with overall reasoning performance, such that increased efficiency correlated with better task performance $(r=0.33, p<0.01)$. Results are visualized at $15 \%$ network density.

\section{Within-module global efficiency and behavior}

Our final analysis sought to investigate changes in global efficiency within each major module evident during the task. Global efficiency has previously been taken to be an index of increased capacity for information exchange (Achard and Bullmore, 2007). Specifically, we were interested in whether each module showed changes in efficiency, and whether any such changes were related to reasoning performance.

Nonparametric Friedman tests revealed that both the sensory (Fig. 5a, orange) and FPV modules (Fig. 5a, green) demonstrated significant differences in efficiency across conditions (sensory: $\chi^{2}=44.17, p<0.001$; FPV $: \chi^{2}=77.78, p<0.001$; Fig. $5 a$ ). No such effect was found for the default-mode module $(p=0.23)$. Bonferroni-corrected follow-up tests confirmed that the effect was driven by increased efficiency within all task states compared with Pre-task and Post-task resting-states (sensory: $z$ range $=$ $2.4-4.69, p<0.02$; FPV: $z$ range $=3.78-5.22, p<0.001$ ).

We also investigated the relationship between individual differences in module efficiency and behavioral performance. To do so, we correlated reasoning accuracy scores with changes in module efficiency between the Pre-task resting-state and the most complex reasoning condition (Quaternary). Only module efficiency within the FPV module was significantly correlated with behavior (Fig. 5b; mean statistics across thresholds: $r=0.33, p=$ 0.026 ; Spearman's $r$ 's $=0.27, p=0.084$ ), such that larger in- creases in efficiency within the FPV module were associated with better reasoning performance. Neither the default-mode or sensory modules demonstrated such a relationship $(p=0.19, p=0.29$, respectively). Further, to probe the reliability of the above finding, we compared change in efficiency from Quaternary to the Nulltask state, which yielded a similar result ( $r=0.35, p=0.021$, Spearman's $r$ 's $=$ $0.30, p=0.048)$. The correlation was also robust to partialling out fluid intelligence scores based on the Raven's Matrices test $(r=0.35, p=0.025)$. By contrast, there was no correlation between performance in the visual search task and module efficiency $(N=43, p=0.65)$, suggesting the module efficiency-behavior relationships were specific to the LST. Finally, we also replicated these results, as well as the follow up VIn results (Fig. 3d), using the original visual, sensory, default-mode, and frontoparietal networks defined by Power and colleagues (2011), instead of our own data-driven modules.

\section{Discussion}

Human reasoning has a quantifiable capacity limit (Halford et al., 1998). This limit is thought to arise from the brain's ability to reconfigure interactions between spatially distributed networks (Cocchi et al., 2014; Parkin et al., 2015; Schultz and Cole, 2016), but recent work has highlighted the circumscribed nature of such interactions when compared with whole-brain "resting” architecture (Cole et al., 2014). In light of these recent findings, we examined how global and selective network properties change from resting to reasoning states, and how such changes relate to reasoning behavior. We found that complexitybased limits in reasoning ability rely on selective patterns of connectivity that emerge in addition to a more general task-induced functional architecture.

We used a nonverbal reasoning task, originally designed to test predictions from relational complexity theory, to systematically manipulate reasoning complexity (Halford et al., 1998; Birney et al., 2006; Birney and Bowman, 2009). In doing so, we replicated previous behavioral results by demonstrating a reliable reduction in accuracy and an increase in reaction time as a function of increased complexity (Birney et al., 2006; Zhang et al., 2009; Zeuch et al., 2011). Importantly, an analysis of participants' trialby-trial ratings indicated that task errors were related to complexity demands and not such factors as transitory lapses in attention or disengagement from the task. The behavioral results also confirmed previous reports that individual reasoning capacity limits are correlated with scores on standard measures of fluid intelligence (Birney et al., 2006; Bhandari and Duncan, 2014), such as Raven's Matrices.

Parametric increases in relational complexity have previously been tied to neural activity of segregated regions of the prefrontal cortices (Christoff et al., 2001; Kroger et al., 2002; Bunge et al., 2009; Golde et al., 2010), as well as to functional connectivity 
a

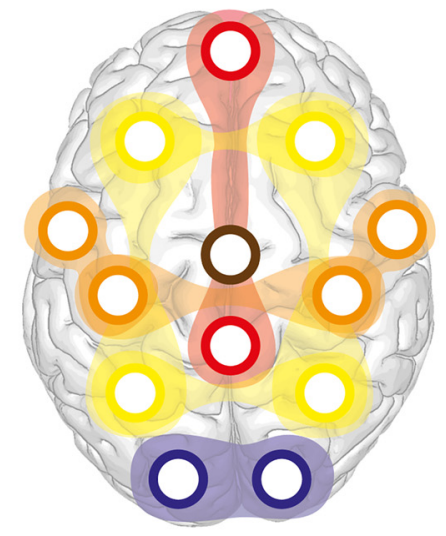

b

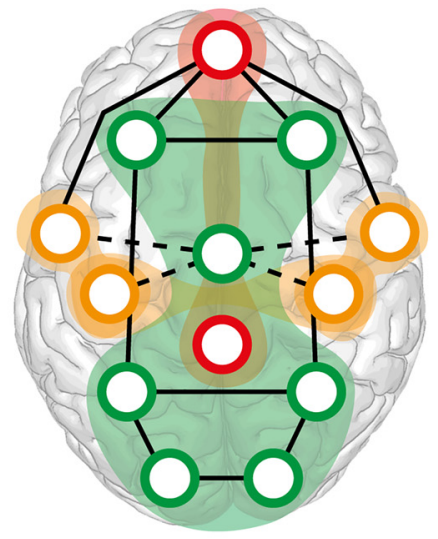

c

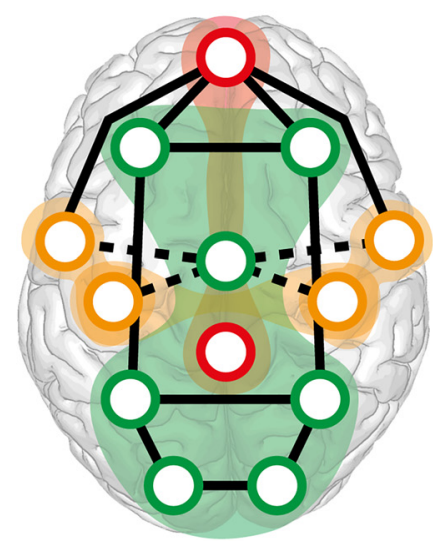

Figure 6. Conceptual model of functional networks supporting reasoning and rest states. $\boldsymbol{a}$, At rest, functional modules are relatively independent. $\boldsymbol{b}$, External, goal-directed task states are accompanied by broad module-level changes; a FPV module forms (green), among stable default-mode (red) and sensory-motor modules (orange). c, Increased task demands are accompanied by specific increases (solid lines) and decreases (dashed lines) in functional connectivity, rather than further modular reconfiguration. Ultimately, in the most complex conditions, the entire network reaches a similar level of correlation through both integrated and segregated dynamics (Fig. $4 b$ ).

within frontoparietal and cingulo-opercular "multiple-demand" networks (Cocchi et al., 2014; Parkin et al., 2015; Crittenden et al., 2016). Cingulo-opercular connectivity has been associated with initiating and maintaining task sets (Dosenbach et al., 2006), whereas the frontoparietal network has been associated with moment-to-moment cognitive control (Cole and Schneider, 2007). Here we found that reasoning performance was best explained by a module composed of brain regions within the frontoparietal, salience, subcortical, and visual networks. First, functional connectivity and global efficiency of this subnetwork increased in line with increased reasoning demands (Figs. 4, 5). Second, larger increases in global efficiency within this module were associated with higher accuracy in the reasoning task. Finally, edge-wise connectivity of the frontoparietal network was shown to increase in line with relational complexity, largely between default-mode and subcortical networks. These findings are broadly consistent with previous work showing that enhanced global network efficiency, and connectivity within the default-mode and frontoparietal networks at rest, can predict intelligence and reasoning performance (Song et al., 2008, 2009; van den Heuvel et al., 2009; Finn et al., 2015; Hearne et al., 2016). Together, the results confirm the central role of flexible frontoparietal connectivity in implementing external goal-directed cognitive control (Cole et al., 2013; Cocchi et al., 2014).

It has been proposed that the cingulo-opercular network can be further divided to include a separate "salience" system associated with bottom-up attention (Seeley et al., 2007; Power et al., 2011). Here we found that the salience network was implicated in the FPV module but did not show complexity-induced edge-wise connectivity changes. On the other hand, the cingulo-opercular network did show edge-wise connectivity changes in line with reasoning complexity, but was not implicated in the FPV module. This set of results is consistent with the notion that the cinguloopercular network is a control-related counterpart of the frontoparietal network, and suggests that the cingulo-opercular network might have a distinct role from that of the salience aspect of the system (Power et al., 2011).

It remains unclear precisely how "resting-state" networks coordinate flexible patterns of integration and segregation as a function of task complexity. Nevertheless, our findings support a key role for subcortical structures, such as the thalamus, in me- diating such relationships (Bell and Shine, 2016; Sherman, 2016). Specifically, the bilateral putamen and thalamus were implicated in both subnetworks that increased and decreased functional connectivity as task complexity increased (Fig. 4b). This finding is in line with recent descriptions of the thalamus as a "global kinless" hub, with evidence of activation in multiple cognitive contexts and strong connectivity across multiple large-scale functional networks (Guimera et al., 2006; van den Heuval and Sporns, 2011; Hwang et al., 2017). One might argue that these subcortical regions manage the relationship between task-related networks to form a coherent modular structure. Further work will be needed to elucidate the particular role of subcortical regions in this relatively unexplored area (Bell and Shine, 2016).

Functional brain module reconfigurations have previously been related to performance on a range of higher cognitive tasks, including learning (Bassett et al., 2011), working memory (Braun et al., 2015; Vatansever et al., 2015, 2017), and cognitive control (Dwyer et al., 2014). Here we found that the community architecture of the brain is flexible, but only in response to large cognitive shifts. For example, resting-state visual and frontoparietal modules, each of which is composed of several known subnetworks (Power et al., 2011), merged during the reasoning task (Fig. 3). Importantly, this reorganization was relatively isolated; follow-up analyses indicated that the rest of the brain remained stable across changes in task complexity. In line with this observation, recent network-based reconceptualizations of global workspace theory (Dehaene et al., 1998; Kitzbichler et al., 2011) have suggested that large, task-based module reconfigurations arise to better serve network communication underpinning behavior. Our work refines this idea by showing that once resting-state modules reconfigure in response to external task demands, most connectivity changes occur without interrupting the newly established modular architecture, as illustrated schematically in Figure 6. Moreover, it is these connectivity changes within the newly reconfigured modules that seem to be most related to behavior.

Our finding that increased demands on cognitive reasoning are paralleled by a reduction in network modularity and increased efficiency has now been reported in several different task contexts (Kitzbichler et al., 2011; Bola and Sabel, 2015; Godwin et al., 2015; Vatansever et al., 2015; Cohen and D'Esposito, 2016; 
Shine et al., 2016; Westphal et al., 2017; Yue et al., 2017). Using the NBS, we found that these global changes were supported by increases and decreases in functional connectivity across multiple large-scale networks. Interestingly, the default-mode network has been suggested to act as a "global integrator," facilitating frontoparietal cognitive control networks during conscious processing of information (Dehaene et al., 1998; Guldenmund et al., 2012; Leech et al., 2012; Vatansever et al., 2015). In line with this notion, we found that default-mode regions, such as the medial frontal cortex, angular gyri, and posterior cingulate cortex, demonstrated increased functional connectivity with frontoparietal, cingulo-opercular, and visual networks as task demands increased.

Modulations of visual network connectivity might be related to the visual nature of the LST, and specifically the requirement that participants search the $4 \times 4$ matrix to identify a shape at the probed location. A previous behavioral study found that participants' eye-fixation patterns differed for one-object and twoobject relational problems (Gordon and Moser, 2007), raising the possibility that changes in relational complexity might be associated with changes in search patterns (and by extension, associated network connectivity). We cannot unequivocally rule out potentially small differences in eye-movement patterns between complexity conditions in our study, but there are at least two reasons why such findings are unlikely to be directly relevant here. First, Gordon and Moser (2007) actively encouraged visual search by having their participants compare two different picture stimuli arranged one above the other on a page. By contrast, our LST paradigm involved the relatively brief presentation of a single $4 \times 4$ matrix at fixation. Second, the stimuli used by Gordon and Moser (2007) were visually complex line drawings that included several different object types that varied in size, shape, and semantic content across trials. By contrast, our LST stimuli involved a single matrix containing identical shapes across complexity conditions, and did not explicitly require active search to solve for the target. Finally, we found no correlation between performance on a standard visual search task and reasoning performance or brain-based network efficiency metrics (subset of participants, $N=43$ ). If increasing visual search demands across task conditions was responsible for the observed network differences, performance on this visual search task should have correlated with the brain-derived metrics.

In conclusion, our findings suggest that reasoning demands rely on selective patterns of connectivity within frontoparietal, salience, cingulo-opercular, subcortical, and default-mode networks, which emerge in addition to a more general, task-induced modular architecture. Further work will be needed to elucidate the network processes that bring about the intricate and coordinated changes in connectivity patterns at the level of edges, modules, and the whole brain, in the service higher cognition. Meanwhile, the current results provide novel insights into the roles of both specific and global network changes in reasoning.

\section{References}

Achard S, Bullmore E (2007) Efficiency and cost of economical brain functional networks. PLoS Comput Biol 3:e17. CrossRef Medline

Ashburner J (2007) A fast diffeomorphic image registration algorithm. Neuroimage 38:95-113. CrossRef Medline

Bassett DS, Bullmore ET, Meyer-Lindenberg A, Apud JA, Weinberger DR, Coppola R (2009) Cognitive fitness of cost-efficient brain functional networks. Proc Natl Acad Sci U S A 106:11747-11752. CrossRef Medline

Bassett DS, Wymbs NF, Porter MA, Mucha PJ, Carlson JM, Grafton ST (2011) Dynamic reconfiguration of human brain networks during learning. Proc Natl Acad Sci U S A 108:7641-7646. CrossRef Medline

Behzadi Y, Restom K, Liau J, Liu TT (2007) A component based noise correction method (CompCor) for BOLD and perfusion based fMRI. Neuroimage 37:90-101. CrossRef Medline
Bell PT, Shine JM (2016) Subcortical contributions to large-scale network communication. Neurosci Biobehav Rev 71:313-322. CrossRef Medline

Bhandari A, Duncan J (2014) Goal neglect and knowledge chunking in the construction of novel behaviour. Cognition 130:11-30. CrossRef Medline

Birney DP, Bowman DB (2009) An experimental-differential investigation of cognitive complexity. Psychol Sci Q 51:449-469.

Birney DP, Halford GS, Andrews G (2006) Measuring the influence of complexity on relational reasoning: the development of the Latin Square Task. Educ Psychol Meas 66:146-171. CrossRef

Blondel VD, Guillaume J-L, Lambiotte R, Lefebvre. E (2008) Fast unfolding of communities in large networks. J Stat Mech Theory Exp 2008:P10008. CrossRef

Bola M, Sabel BA (2015) Dynamic reorganization of brain functional networks during cognition. Neuroimage 114:398-413. CrossRef Medline

Bolt T, Nomi JS, Rubinov M, Uddin LQ (2017) Correspondence between evoked and intrinsic functional brain network configurations. Hum Brain Mapp 38:1992-2007. CrossRef Medline

Braun U, Schäfer A, Walter H, Erk S, Romanczuk-Seiferth N, Haddad L, Schweiger JI, Grimm O, Heinz A, Tost H, Meyer-Lindenberg A, Bassett DS (2015) Dynamic reconfiguration of frontal brain networks during executive cognition in humans. Proc Natl Acad Sci U S A 112:1167811683. CrossRef Medline

Bunge SA, Helskog EH, Wendelken C (2009) Left, but not right, rostrolateral prefrontal cortex meets a stringent test of the relational integration hypothesis. Neuroimage 46:338-342. CrossRef Medline

Caballero-Gaudes C, Reynolds RC (2017) Methods for cleaning the BOLD fMRI signal. Neuroimage 154:128-149. CrossRef Medline

Chao-Gan Y, Yu-Feng Z (2010) DPARSF: a MATLAB toolbox for "pipeline" data analysis of resting-State fMRI. Front Syst Neurosci 4:13. CrossRef Medline

Christoff K, Prabhakaran V, Dorfman J, Zhao Z, Kroger JK, Holyoak KJ, Gabrieli JD (2001) Rostrolateral prefrontal cortex involvement in relational integration during reasoning. Neuroimage 14:1136-1149. CrossRef Medline

Ciric R, Wolf DH, Power JD, Roalf DR, Baum GL, Ruparel K, Shinohara RT, Elliott MA, Eickhoff SB, Davatzikos C, Gur RC, Gur RE, Bassett DS, Satterthwaite TD (2017) Benchmarking of participant-level confound regression strategies for the control of motion artifact in studies of functional connectivity. Neuroimage 154:174-187. CrossRef Medline

Cocchi L, Zalesky A, Fornito A, Mattingley JB (2013) Dynamic cooperation and competition between brain systems during cognitive control. Trends Cogn Sci 17:493-501. CrossRef Medline

Cocchi L, Halford GS, Zalesky A, Harding IH, Ramm BJ, Cutmore T, Shum DH, Mattingley JB (2014) Complexity in relational processing predicts changes in functional brain network dynamics. Cereb Cortex 24:22832296. CrossRef Medline

Cocchi L, Yang Z, Zalesky A, Stelzer J, Hearne LJ, Gollo LL, Mattingley JB (2017) Neural decoding of visual stimuli varies with fluctuations in global network efficiency. Hum Brain Mapp 38:3069-3080. CrossRef Medline

Cohen JR, D'Esposito M (2016) The segregation and integration of distinct brain networks and their relationship to cognition. J Neurosci 36:1208312094. CrossRef Medline

Cole MW, Schneider W (2007) The cognitive control network: integrated cortical regions with dissociable functions. Neuroimage 37:343-360. CrossRef Medline

Cole MW, Reynolds JR, Power JD, Repovs G, Anticevic A, Braver TS (2013) Multi-task connectivity reveals flexible hubs for adaptive task control. Nat Neurosci 16:1348-1355. CrossRef Medline

Cole MW, Bassett DS, Power JD, Braver TS, Petersen SE (2014) Intrinsic and task-evoked network architectures of the human brain. Neuron 83: 238-251. CrossRef Medline

Cole MW, Ito T, Bassett DS, Schultz DH (2016) Activity flow over restingstate networks shapes cognitive task activations. Nat Neurosci 19:17181726. CrossRef Medline

Crittenden BM, Mitchell DJ, Duncan J (2016) Task encoding across the multiple demand cortex is consistent with a frontoparietal and cinguloopercular dual networks distinction. J Neurosci 36:6147-6155. CrossRef Medline

Crossley NA, Mechelli A, Vértes PE, Winton-Brown TT, Patel AX, Ginestet CE, McGuire P, Bullmore ET (2013) Cognitive relevance of the commu- 
nity structure of the human brain functional coactivation network. Proc Natl Acad Sci U S A 110:11583-11588. CrossRef Medline

Dehaene S, Kerszberg M, Changeux JP (1998) A neuronal model of a global workspace in effortful cognitive tasks. Proc Natl Acad Sci U S A 95: 14529-14534. CrossRef Medline

de Pasquale F, Della Penna S, Sporns O, Romani GL, Corbetta M (2016) A dynamic core network and global efficiency in the resting human brain. Cereb Cortex 26:4015-4033. CrossRef Medline

Dosenbach NU, Visscher KM, Palmer ED, Miezin FM, Wenger KK, Kang HC, Burgund ED, Grimes AL, Schlaggar BL, Petersen SE (2006) A core system for the implementation of task sets. Neuron 50:799-812. CrossRef Medline

Dwyer DB, Harrison BJ, Yücel M, Whittle S, Zalesky A, Pantelis C, Allen NB, Fornito A (2014) Large-scale brain network dynamics supporting adolescent cognitive control. J Neurosci 34:14096-14107. CrossRef Medline

Finn ES, Shen X, Scheinost D, Rosenberg MD, Huang J, Chun MM, Papademetris X, Constable RT (2015) Functional connectome fingerprinting: identifying individuals using patterns of brain connectivity. Nat Neurosci 18:1664-1671. CrossRef Medline

Fortunato S (2010) Community detection in graphs. Phys Rep 486:75-174. CrossRef

Garrison KA, Scheinost D, Finn ES, Shen X, Constable RT (2015) The (in)stability of functional brain network measures across thresholds. Neuroimage 118:651-661. CrossRef Medline

Gerchen MF, Bernal-Casas D, Kirsch P (2014) Analyzing task-dependent brain network changes by whole-brain psychophysiological interactions: a comparison to conventional analysis. Hum Brain Mapp 35:5071-5082. CrossRef Medline

Godwin D, Barry RL, Marois R (2015) Breakdown of the brain's functional network modularity with awareness. Proc Natl Acad Sci U S A 112:3799_ 3804. CrossRef Medline

Golde M, von Cramon DY, Schubotz RI (2010) Differential role of anterior prefrontal and premotor cortex in the processing of relational information. Neuroimage 49:2890-2900. CrossRef Medline

Good BH, de Montjoye Y-AA, Clauset A (2010) Performance of modularity maximization in practical contexts. Phys Rev E Stat Nonlin Soft Matter Phys 81:46106. CrossRef Medline

Gordon PC, Moser S (2007) Insight into analogies: evidence from eye movements. Vis Cogn 15:20-35. CrossRef Medline

Guimera R, Sales-Pardo M, Amaral LAN (2006) Classes of complex networks defined by role-to-role connectivity profiles. Nat Phys 3:63-69. CrossRef

Guldenmund P, Vanhaudenhuyse A, Boly M, Laureys S, Soddu A (2012) A default mode of brain function in altered states of consciousness. Arch Ital Biol 150:107-121. CrossRef Medline

Halford GS, Wilson WH, Phillips S (1998) Processing capacity defined by relational complexity: implications for comparative, developmental, and cognitive psychology. Behav Brain Sci 21:803-831; discussion 831-864. Medline

Halford GS, Baker R, McCredden JE, Bain JD (2005) How many variables can humans process? Psychol Sci 16:70-76. CrossRef Medline

Hearne L, Cocchi L, Zalesky A, Mattingley JB (2015) Interactions between default mode and control networks as a function of increasing cognitive reasoning complexity. Hum Brain Mapp 36:2719-2731. CrossRef Medline

Hearne LJ, Mattingley JB, Cocchi L (2016) Functional brain networks related to individual differences in human intelligence at rest. Sci Rep 6:32328. CrossRef Medline

Hwang K, Bertolero MA, Liu WB, D’Esposito M (2017) The human thalamus is an integrative hub for functional brain networks. J Neurosci 37:55945607. CrossRef

Johnson-Laird PN (2010) Mental models and human reasoning. Proc Natl Acad Sci U S A 107:18243-18250. CrossRef Medline

Kitzbichler MG, Henson RN, Smith ML, Nathan PJ, Bullmore ET (2011) Cognitive effort drives workspace configuration of human brain functional networks. J Neurosci 31:8259-8270. CrossRef Medline

Knowlton BJ, Morrison RG, Hummel JE, Holyoak KJ (2012) A neurocomputational system for relational reasoning. Trends Cogn Sci 16:373-381. CrossRef Medline

Krienen FM, Yeo BTT, Buckner RL (2014) Reconfigurable task-dependent functional coupling modes cluster around a core functional architecture. Philos Trans R Soc Lond B Biol Sci 369:pii:20130526. CrossRef Medline

Kroger JK, Sabb FW, Fales CL, Bookheimer SY, Cohen MS, Holyoak KJ (2002) Recruitment of anterior dorsolateral prefrontal cortex in human reasoning: a parametric study of relational complexity. Cereb Cortex 12: 477-485. CrossRef Medline

Lancichinetti A, Fortunato S (2012) Consensus clustering in complex networks. Sci Rep 2:336. CrossRef Medline

Latora V, Marchiori M (2001) Efficient behavior of small-world networks. Phys Rev Lett 87:198701. CrossRef Medline

Lee IA, Preacher KJ (2013) Calculation for the test of the difference between two dependent correlations with one variable in common [computer software]. Available at http://quantpsy.org/.

Leech R, Braga R, Sharp DJ (2012) Echoes of the brain within the posterior cingulate cortex. J Neurosci 32:215-222. CrossRef Medline

McLaren DG, Ries ML, Xu G, Johnson SC (2012) A generalized form of context-dependent psychophysiological interactions (gPPI): a comparison to standard approaches. Neuroimage 61:1277-1286. CrossRef Medline

Meilă M (2007) Comparing clusterings-an information based distance. J Multivar Anal 98:873-895. CrossRef

Mill RD, Ito T, Cole MW (2017) From connectome to cognition: the search for mechanism in human functional brain networks. Neuroimage pii: S1053-8119(17)30083-6. CrossRef Medline.

Moeller S, Yacoub E, Olman C a, Auerbach E, Strupp J, Harel N, Ugǔrbil K (2010) Multiband multislice GE-EPI at 7 tesla, with 16-fold acceleration using partial parallel imaging with application to high spatial and temporal whole-brain FMRI. Magn Reson Med 63:1144-1153. CrossRef Medline

Newman ME, Girvan M (2004) Finding and evaluating community structure in networks. Phys Rev E Stat Nonlin Soft Matter Phys 69:026113. Medline

Newman ME, Strogatz SH, Watts DJ (2001) Random graphs with arbitrary degree distributions and their applications. Phys Rev E Stat Nonlin Soft Matter Phys 64:026118. CrossRef Medline

Parkin BL, Hellyer PJ, Leech R, Hampshire A (2015) Dynamic network mechanisms of relational integration. J Neurosci 35:7660-7673. CrossRef Medline

Penn DC, Holyoak KJ, Povinelli DJ (2008) Darwin's mistake: explaining the discontinuity between human and nonhuman minds. Behav Brain Sci 31:109-130; discussion 130-178. CrossRef Medline

Power JD, Cohen AL, Nelson SM, Wig GS, Barnes KA, Church JA, Vogel AC, Laumann TO, Miezin FM, Schlaggar BL, Petersen SE (2011) Functional network organization of the human brain. Neuron 72:665-678. CrossRef Medline

Power JD, Mitra A, Laumann TO, Snyder AZ, Schlaggar BL, Petersen SE (2014) Methods to detect, characterize, and remove motion artifact in resting state fMRI. Neuroimage 84:320-341. CrossRef Medline

Raven J (2000) The Raven's progressive matrices: change and stability over culture and time. Cogn Psychol 41:1-48. CrossRef Medline

Rosvall M, Axelsson D, Bergstrom CT (2009) The map equation. Eur Phys J Spec Top 178:13-23. CrossRef

Rubinov M, Sporns O (2010) Complex network measures of brain connectivity: uses and interpretations. Neuroimage 52:1059-1069. CrossRef Medline

Rubinov M, Sporns O (2011) Weight-conserving characterization of complex functional brain networks. Neuroimage 56:2068-2079. CrossRef Medline

Saad ZS, Gotts SJ, Murphy K, Chen G, Jo HJ, Martin A, Cox RW (2012) Trouble at rest: how correlation patterns and group differences become distorted after global signal regression. Brain Connect 2:25-32. CrossRef Medline

Schultz DH, Cole MW (2016) Higher intelligence is associated with less task-related brain network reconfiguration. J Neurosci 36:8551-8561. CrossRef Medline

Seeley WW, Menon V, Schatzberg AF, Keller J, Glover GH, Kenna H, Reiss AL, Greicius MD (2007) Dissociable intrinsic connectivity networks for salience processing and executive control. J Neurosci 27:2349-2356. CrossRef Medline

Sherman SM (2016) Thalamus plays a central role in ongoing cortical functioning. Nat Neurosci 16:533-541. CrossRef Medline

Shine JM, Bissett PG, Bell PT, Koyejo O, Balsters JH, Gorgolewski KJ, Moodie CA, Poldrack RA (2016) The dynamics of functional brain networks: integrated network states during cognitive task performance. Neuron 92 : 544-554. CrossRef Medline

Smallwood J, Schooler JW (2015) The science of mind wandering: empiri- 
cally navigating the stream of consciousness. Annu Rev Psychol 66:487518. CrossRef Medline

Song M, Zhou Y, Li J, Liu Y, Tian L, Yu C, Jiang T (2008) Brain spontaneous functional connectivity and intelligence. Neuroimage 41:1168-1176. CrossRef Medline

Song M, Liu Y, Zhou Y, Wang K, Yu C, Jiang T (2009) Default network and intelligence difference. Conf Proc IEEE Eng Med Biol Sci 2009:22122215. CrossRef Medline

Sporns O, Betzel RF (2016) Modular brain networks. Annu Rev Psychol 67:613-640. CrossRef Medline

Sun FT, Miller LM, D’Esposito M (2004) Measuring interregional functional connectivity using coherence and partial coherence analyses of fMRI data. Neuroimage 21:647-658. CrossRef Medline

Tavor I, Parker Jones O, Mars RB, Smith SM, Behrens TE, Jbabdi S (2016) Task-free MRI predicts individual differences in brain activity during task performance. Science 352:216-220. CrossRef Medline

Treisman AM, Gelade G (1980) A feature-integration theory of attention. Cogn Psychol 12:97-136. CrossRef Medline

van den Heuvel MP, Stam CJ, Kahn RS, Hulshoff Pol HE (2009) Efficiency of functional brain networks and intellectual performance. J Neurosci 29:7619-7624. CrossRef Medline

van den Heuvel MP, Sporns O (2011) Rich-club organization of the human connectome. J Neurosci 31:15775-15786. CrossRef Medline

Van Dijk KR, Sabuncu MR, Buckner RL (2012) The influence of head mo- tion on intrinsic functional connectivity MRI. Neuroimage 59:431-438. CrossRef Medline

Vatansever D, Menon DK, Manktelow AE, Sahakian BJ, Stamatakis EA (2015) Default mode dynamics for global functional integration. J Neurosci 35:15254-15262. CrossRef Medline

Vatansever D, Manktelow AE, Sahakian BJ, Menon DK, Stamatakis EA (2017) Angular default mode network connectivity across working memory load. Hum Brain Mapp 38:41-52. CrossRef Medline

Westphal AJ, Wang S, Rissman J (2017) Episodic memory retrieval benefits from a less modular brain network organization. J Neurosci 37:35233531. CrossRef Medline

Yue Q, Martin RC, Fischer-Baum S, Ramos-Nuñez AI, Ye F, Deem MW (2017) Brain modularity mediates the relation between task complexity and performance. J Cogn Neurosci 29:1532-1546. CrossRef Medline

Zalesky A, Fornito A, Bullmore ET (2010) Network-based statistic: identifying differences in brain networks. Neuroimage 53:1197-1207. CrossRef Medline

Zalesky A, Fornito A, Bullmore E (2012) On the use of correlation as a measure of network connectivity. Neuroimage 60:2096-2106. CrossRef Medline

Zeuch N, Holling H, Kuhn J-T (2011) Analysis of the Latin Square Task with linear logistic test models. Learn Individ Differ 21:629-632. CrossRef

Zhang L, Xin Z, Lin C, Li H (2009) The complexity of the Latin Square Task and its influence on children's performance. Chinese Sci Bull 54:766-775. 